\title{
A Note on applications of q-Theory
}

\author{
Prashant Singh ${ }^{1}$, Pramod Kumar Mishra ${ }^{2}$ \\ ${ }^{1}$ (Department of Computer Science, Banaras Hindu University, India) \\ ${ }^{2}$ (Deparment of Computer Science, Banaras Hindu University, India)
}

\begin{abstract}
This paper deals with describing application of q-theory in different fields of mathematics and future areas where its use can be extended.

Keywords: q-analogue, q-function, q-hypergeometric function

\section{Introduction}

Basic hypergeometric series are called $q$-analogues (basic analogues or $q$-extensions) of hypergeometric series.

q-hypergeometric series, are q-analog generalizations of generalized hypergeometric series, and are in turn generalized by elliptic hypergeometric series. A series $x_{n}$ is called hypergeometric if the ratio of successive terms $x_{n+1} / x_{n}$ is a rational function of $n$. If the ratio of successive terms is a rational function of $q^{n}$, then the series is called a basic hypergeometric series. The number $q$ is called the base or parameter which lies between 0 and 1.Value of q determines accuracy of analogue of any classical function.
\end{abstract}

\subsection{Basic Differentiation operator}

$D_{q, x} f(x)=\frac{f(q x)-f(x)}{x(q-1)}$

1.2 q-Integration

$\int_{a}^{b} f(x) d q x=(1-q)\left\{b \sum_{r=0}^{\infty} q^{r} f\left(q^{r} b\right)-a \sum_{r=0}^{\infty} q^{r} f\left(q^{r} a\right)\right\}$

1.3 q-exponential function

A whole family of q exponential function can be defined as

$\mathrm{E}(\mathrm{q}, \beta ; \mathrm{x})=\sum_{r=0}^{\infty} x^{r} q^{\beta \mathrm{r}(\mathrm{r}-1)} /[r ; q]$ !

depending upon value of $\beta$ i.e. $\beta=0, \beta=1 / 2$ and $\beta=1 / 4$ repectively.

\subsection{Forward Differences}

\section{II. q-analogue of some statistical functions}

If $\mathrm{y}_{0}, \mathrm{y}_{1}, \mathrm{y}_{2}, \ldots, \mathrm{yn}$ denote a set of values of $\mathrm{y}$, where $\mathrm{y}=\mathrm{f}(\mathrm{z})$, then $\mathrm{y}_{1}-\mathrm{y}_{0}, \mathrm{y}_{2}-\mathrm{y}_{1}, \ldots, \mathrm{y}_{\mathrm{n}}-\mathrm{y}_{\mathrm{n}-1}$ are called differences of $\mathrm{y}$, where $\Delta$ is called forward difference operator and $\nabla$ is called backward difference operator and as $0<\mathrm{q}<1$, $\mathrm{f}(\mathrm{z})>\mathrm{f}(\mathrm{qz})$.

$\Delta f(z)=f(z)-f(q z)$

$\Delta^{2} f(z)=\Delta f(z)-\Delta f(q z)=f\left(q^{2} z\right)-2 f(q z)+f(z)$

$\Delta^{3} f(z)=f\left(q^{3} z\right)-3 f\left(q^{2} z\right)+3 f(q z)-f(z)$

$\Delta^{4} f(z)=f\left(q^{4} z\right)-4 f\left(q^{3} z\right)+6 f\left(q^{2} z\right)-4 f(q z)+f(z)$

\subsection{Backward Differences}

$\nabla f(q z)=f(q z)-f(z)$

$\nabla f\left(q^{2} z\right)=f\left(q^{2} z\right)-f(q z)$

$\nabla^{2} f\left(q^{2} z\right)=\nabla f\left(q^{2} z\right)-\nabla f(q z)=f\left(q^{2} z\right)-2 f(q z)+f(z)$

$\nabla^{3} f\left(q^{3} z\right)=f\left(q^{3} z\right)-3 f\left(q^{2} z\right)+3 f(q z)-f(z)$

\section{3 q -analogue of Moment Generating Function}

Let $\mathrm{X}$ be a random variable.

Then, $\operatorname{EXPEC}\left(E_{q}(t x)\right)=\operatorname{EXPEC}\left(\sum \frac{(t x)^{r}}{[r ; q] !}\right)=1+t \mu_{1}+\frac{t^{2}}{[2 ; q] !} \mu_{2}+\cdots+\frac{t^{r}}{[r ; q] !} \mu_{r}$,

where EXPEC is expected value.

The coefficient of $\frac{t^{r}}{[r ; q] !}$ in the expression is $\mu_{r}$, the $\mathrm{r}^{\text {th }}$ moment of $\mathrm{X}$ about origin. 
MGF for discrete random variable with probability distribution

\begin{tabular}{|lll|}
\hline $\mathrm{X}:$ & $\mathrm{x} 1$ & $\mathrm{x} 2 \ldots \ldots \ldots \ldots \ldots \mathrm{xn}$ \\
\hline $\mathrm{P}(\mathrm{x}):$ & $\mathrm{p} 1$ & $\mathrm{p} 2 \ldots \ldots \ldots \ldots \ldots . \mathrm{pn}$ \\
\hline
\end{tabular}

$M(t)=\operatorname{EXPEC}(E(q, t x))=\sum E_{q}(t X) p_{i}$

\section{MGF for a Continuous Random Variable}

Let $\mathrm{X}$ be a continuous random variable with probability density function $\mathrm{f}(\mathrm{x})$

,$-\infty<x<\infty$

$M(t)=\operatorname{Expec}(E(q, t x))=\int_{-\infty}^{\infty} \boldsymbol{E}(\boldsymbol{q}, \boldsymbol{t} \boldsymbol{x}) \boldsymbol{f}(\boldsymbol{x}) \boldsymbol{d}(\boldsymbol{q} \boldsymbol{x})$

$\operatorname{Expec}(\mathrm{E}(\mathrm{q}, \mathrm{tx}))$ is $\mathrm{mgf}$ about origin.

$\operatorname{Expec}(E(q, t(x-a))$ is mgf about point a.

$\operatorname{Expec}(E(q, t(x-\bar{x}))$ is mgf about origin.

\section{Properties of mgf}

$$
\begin{aligned}
& \operatorname{Expec}(E(q, t(x+y)))=\operatorname{Expec}(E(q, t x))+\operatorname{Expec}(E(q, t y)) \\
& \operatorname{Expec}\left(E_{q}(t(x+y))\right)=\operatorname{Expec}\left(E_{q}(t x)\right)+\operatorname{Expec}\left(E_{q}(t y)\right) \\
& \operatorname{Expec}\left(E_{q}(t(u+c))\right)=E_{q}(t u)+\operatorname{Expec}\left(E_{q}(t c)\right)
\end{aligned}
$$

\section{$2.4 \mathbf{q}$-Distribution Function}

If $F_{q}(x)=\int_{-\infty}^{\infty} f(x) d(q x)=P(X \leq x)$ then the function $F_{q}(x)$ is the probability that the value of the variable will be less or equal to $x$. Thus, $F_{q}(x)=P(X \leq x)$ and

$F_{q}(b)-F_{q}(a)=\int_{a}^{b} f(x) d(q x)=P(a \leq X \leq b) . F_{q}(x)$ is called the cumulative distribution function of $\mathrm{X}$ or simply distribution function.

\section{Properties}

$$
F_{q}(-\infty)=0 \text { and } F_{q}(\infty)=1
$$

\section{5 q- analogue of Differential Equation}

Solution of second order linear differential equation with constant coefficients

$D_{q}^{2} y-a_{1} D_{q}^{1} y+a_{2}=0$

If auxiliary equation has real and distinct roots $\mathrm{m}_{1}$ and $\mathrm{m}_{2}$, general solution is

$y=A E_{q}\left(m_{1} \mathrm{x}\right)+\mathrm{B} E_{q}\left(m_{2} \mathrm{x}\right)$

$y=A E\left(q ; m_{1} \mathrm{x}\right)+\mathrm{BE}\left(q ; m_{2} \mathrm{x}\right)$

$y=A E\left(1 / q ; m_{1} \sqrt{ } q \mathrm{x}\right)+\mathrm{BE}\left(1 / q ; m_{2} \sqrt{q} \mathrm{x}\right)$

Real and equal roots

If $\mathrm{m} 1=\mathrm{m} 2=\mathrm{m}$

$\mathrm{y}=(\mathrm{Ax}+\mathrm{B}) \mathrm{E}_{\mathrm{q}}(\mathrm{mx})$ or $\mathrm{y}=(\mathrm{Ax}+\mathrm{B}) \mathrm{E}(\mathrm{q} ; \mathrm{m} \sqrt{q x})$ or $\mathrm{y}=(\mathrm{Ax}+\mathrm{B}) \mathrm{E}(1 / \mathrm{q} ; \mathrm{m} \sqrt{q x})$

Complex Conjugate Roots

If $\alpha$ and $\beta$ be the real and imaginary parts of the roots then general solution will take form

$y=C 1 E(q ; \alpha \sqrt{q x}) \sin (q ;(\beta \mathrm{x}+\mathrm{C} 2)$

\subsection{Basic Analogue of Integral Transforms}

\subsection{1 q-Laplace Transform}

$f(s)=\int_{0}^{\infty} F(t) E(q,-s t) d(q t)$ 


\subsection{2 q-Fourier Transform}

$f(s)=\int_{-\infty}^{\infty} F(t) E(q,-i s t) d(q t)$

\subsection{3q-MellinTransform}

$f(s)=\int_{0}^{\infty} F(t) t^{s-1} d(q t)$

\subsection{4 q-HankelTransform}

$$
f(s)=\int_{0}^{\infty} F(t) t J_{n}(s t) d(q t)
$$

\subsection{Basic Analogue of Newton Cotes Integration}

$\mathrm{I}=\int_{a}^{b} w(x) f(x) d q x=\sum_{k=0}^{n} m_{k} f\left(x_{k}\right)$ where $\mathrm{x}_{1}, \mathrm{x}_{2}, \ldots, \mathrm{x}_{\mathrm{n}}$ are nodes distributed within limits of integration.

$R_{n}=(1-q)\left\{b \sum_{r=0}^{\infty} q^{r} w\left(q^{r} b\right) f\left(q^{r} b\right)-a \sum_{r=0}^{\infty} q^{r} w\left(q^{r} a\right) f\left(q^{r} a\right)\right\}-\sum_{k=0}^{n} m_{k} f\left(x_{k}\right)$

$R_{n}$ is the error term.

If $\mathrm{w}(\mathrm{x})=1$ and nodes $\mathrm{x}_{\mathrm{k}}{ }^{\mathrm{s}}$ are distributed in $[\mathrm{a}, \mathrm{b}]$ with $\mathrm{x}_{0}=\mathrm{a}, \mathrm{x}_{\mathrm{n}}=\mathrm{b}$ and $\mathrm{h}=(\mathrm{b}-\mathrm{a}) / \mathrm{n}, \mathrm{x}_{\mathrm{k}}=\mathrm{x}_{0}+\mathrm{kh}, \mathrm{k} \in \mathrm{n}$.

$(1-q)\left\{b \sum_{r=0}^{\infty} q^{r} w\left(q^{r} b\right) f\left(q^{r} b\right)-a \sum_{r=0}^{\infty} q^{r} w\left(q^{r} a\right) f\left(q^{r} a\right)\right\}=\frac{(b-a)[f(a)+f(b)]}{[2 ; q] !}$

where $\frac{(b-a)}{[2 ; q] !}=h$.

By putting $\mathrm{w}(\mathrm{x})=1, \mathrm{n}=1, \mathrm{f}(\mathrm{x})=\mathrm{x}$ in $\int_{a}^{b} w(x) f(x) d q x=\sum_{k=0}^{n} m_{k} f\left(x_{k}\right)$ we get,

$m_{0}=m_{1}=\frac{(b-a)}{1+q}$

$\int_{a}^{b} w(x) f(x) d q x=\frac{(b-a)}{[2 ; q] !}(f(a)+f(b))$

which is analogue of Trapezoidal Rule.

$$
\begin{aligned}
\mathrm{R}_{\mathrm{n}} & =\frac{-h^{3}}{12} D_{q}^{2} f(\xi) \\
& =\frac{-h^{3}}{12 q^{2}(q-1)^{2}}\left(f\left(q^{2} \xi\right)-[2 ; q] f(q \xi)+q f(\xi)\right] \\
& =\frac{-h^{3}}{12} \frac{\left[q_{2} f\left(q_{1}^{2} \xi\right)+q_{1} f\left(q_{2}^{2} \xi\right)-q_{1} \mathrm{f}\left(q_{1} q_{2} \xi\right)-q_{2} \mathrm{f}\left(q_{1} q_{2} \xi\right)\right]}{\left(q_{1}-q_{2}\right)^{2} \xi^{2}}
\end{aligned}
$$

By putting $n=2$ and $n=3$ we can easily get analogue for Simpson's $1 / 3$ and Simpsons's $3 / 8$ rule.

\subsection{1 q-Simpson's $1 / 3$ Rule}

By putting $\mathrm{w}(\mathrm{x})=1, \mathrm{n}=2, \mathrm{f}(\mathrm{x})=\mathrm{x}^{2}$ in $\int_{a}^{b} w(x) f(x) d q x=\sum_{k=0}^{n} m_{k} f\left(x_{k}\right)$

we get,

$$
m_{0}=\frac{(b-a)}{[3 ; q]}, m_{1}=\frac{4(b-a)}{[3 ; q]}, m_{2}=\frac{(b-a)}{[3 ; q]}
$$

\subsection{2 q-Simpsons's 3/8 Rule}

By putting $\mathrm{w}(\mathrm{x})=1, \mathrm{n}=3, \mathrm{f}(\mathrm{x})=\mathrm{x}^{3} \operatorname{in} \int_{a}^{b} w(x) f(x) d q x=\sum_{k=0}^{n} m_{k} f\left(x_{k}\right)$

we get,

$m_{0}=\frac{3(b-a)}{2[4 ; q]}, m_{1}=\frac{9(b-a)}{2[4 ; q]}, m_{2}=\frac{9(b-a)}{2[4 ; q]}, m_{3}=\frac{3(b-a)}{2[4 ; q]}$

For a method of order $\mathbf{m}$

Error $=R_{n}=\frac{c}{[m+1 ; q] !} D_{q}^{n} f(\xi)$,

where $\mathrm{a}<\xi<\mathrm{b}$

Error terms in Trapezoidal Rule, Simpson's 1/3 Rule, Simpson's 3/8Rule

$E_{\text {trp }}=-\frac{h^{3}}{12} D_{q}^{2} f(\xi), E_{\text {smp } 1 / 3}=-\frac{C h^{4}}{24} D_{q}^{3} f(\xi), E_{\text {smp } 3 / 8}=-\frac{3 h^{5}}{80} D_{q}^{4} f(\xi)$ 
Weights for Newton's Cotes Integration methods when q tends to one.

\begin{tabular}{|l|l|l|l|l|}
\hline $\mathrm{n}$ & $\mathrm{m}_{0}$ & $\mathrm{~m}_{1}$ & $\mathrm{~m}_{2}$ & $\mathrm{~m}_{3}$ \\
\hline 0 & $1 / 2$ & $1 / 2$ & & \\
\hline 1 & $1 / 3$ & $4 / 3$ & $1 / 3$ & \\
\hline 2 & $3 / 8$ & $9 / 8$ & $9 / 8$ & $3 / 8$ \\
\hline
\end{tabular}

\section{8 q-analogue of Lobatto Integration}

$\int_{-1}^{1} f(x) d q x=2(1-q) \sum_{r=0}^{\infty} q^{r} f\left(q^{r}\right)=m_{0} f(-1)+m_{n} f(n)+\sum_{k=1}^{n-1} m_{k} f\left(x_{k}\right)$

For $\mathrm{n}=2, \int_{-1}^{1} f(x) d q x=\frac{1}{1+q+q^{2}}[f(-1)+f(1)+4 f(0)]$

\section{9 q- analogue of Radau Integration}

$\int_{-1}^{1} f(x) d q x=2(1-q) \sum_{r=0}^{\infty} q^{r} f\left(q^{r}\right)=m_{0} f(-1)+\sum_{k=1}^{n} m_{k} f\left(x_{k}\right)$

For $\mathrm{n}=2$

$\int_{-1}^{1} f(x) d q x=\frac{2}{3\left(1+q+q^{2}\right)} f(-1)+\frac{16+\sqrt{6}}{6\left(1+q+q^{2}\right)} f\left(\frac{1-\sqrt{6}}{5}\right)+\frac{16-\sqrt{6}}{6\left(1+q+q^{2}\right)} f\left(\frac{1+\sqrt{6}}{5}\right)$

\section{Predictor Correctors Method}

\subsection{0 q- analogue of Milne's Method}

$y_{4}=y_{0}+h\left[4 f_{0}+16 \frac{\Delta_{q} f_{0}}{[2 ; q]}+\frac{32[2 ; q]-8[3 ; q]}{[2 ; q][3 ; q]} \Delta_{q}^{2} f_{0}+\frac{2}{3} \Delta_{q}^{3} f_{0}\left\{\frac{[2 ; q][3 ; q] 8^{4}-3 * 16^{2}[4 ; q][2 ; q]+32[4 ; q][3 ; q]}{[4 ; q][2 ; q][3 ; q]}\right\}\right]$

\subsection{1 q-analogue of Moultons Method}

$y_{1}=y_{0}+h\left[f_{0}+\frac{\nabla_{q} f_{0}}{[2 ; q]}+\frac{1}{2}\left(\frac{1}{[3 ; q]}+\frac{1}{[2 ; q]}\right) \nabla_{q}^{2} f_{0}+\frac{1}{6} \nabla_{q}^{3} f_{0}\left(\frac{1}{[4 ; q]}+\frac{3}{[3 ; q]}+\frac{2}{[1 ; q]}\right)\right]$

where, $\Delta_{q}^{n}$ is K.Conrad [4] difference operator

$\Delta_{q}^{n}=\left\{\left(E-q^{n-1}\right) \ldots(E-q)(E-1)\right\}$

where $\mathrm{E}(\mathrm{f}(\mathrm{x}))=\mathrm{f}(\mathrm{x}+\mathrm{h})$

\section{Conclusion}

q-analogue of these methods provide an alternate method of solving classical problems where value of q determines the accuracy of result. q-analogue of different transformations can be used in boundary value problems of differential equations as well as in computer problems where parameters play important role .

\section{References:}

[1] G. Gasper and M. Rahman, Basic Hypergeometric Series, CambridgeUniversity Press, Cambridge, 1990.

[2] Thomas Ernst, A method for q-Calculus, Journal of Nonlinear Mathematical Physics, 2003

[3] Prashant Singh, Pramod Kumar Mishra and R.S.Pathak ,q-Iterative Methods, IOSR Journal of Mathematics (IOSR-JM), Volume 8, Issue 6 (Nov. - Dec. 2013), PP 01-06 